\title{
Algebraic Cycles and Algebraic K-Theory
}

\author{
Daniel R. Grayson \\ Columbia University, New York, New York 10027 \\ Communicated by D. A. Buchsbaum \\ Received November 7, 1978
}

\section{INTRODUCTION}

'I'he purpose of this paper is to develop higher algebraic $K$-theory into a tool for understanding algebraic cycles on a variety.

Bloch made the first step: he showed that the group of zero-cycles modulo rational equivalence is $H^{2}\left(X, \mathscr{K}_{2}\right)$ on a nonsingular surface $X$. Gersten reduced the general statement that $H^{n}\left(X, \mathscr{K}_{n}\right)$ is $A^{n}(X)$, the group of codimension $n$-cycles modulo rational equivalence, to a conjecturc which Quillen proved for nonsingular $X$.

Bloch's next idea was the relativization of the situation using $K$-theory. Dcnote the functor $S \rightarrow H^{n}\left(X \times S, \mathscr{K}_{n}\right)$ by $\mathscr{L}^{n}(X)$. The question of representability of this functor is interesting. Witness the result of Bloch [4] which says that, for a nonsingular surface $X$ of characteristic zero, the following statements are equivalent:

(1) The formal completion of $\mathscr{A}^{2}(X)$ at 0 is pro-representable.

(2) The formal completion of $\mathscr{A}^{2}(X)$ at 0 agrees with that of the Albanese variety of $X, \mathrm{Alb}_{X}$.

(3) The geometric genus, $p_{g}=\operatorname{dim} H^{2}\left(X, \mathbb{O}_{X}\right)$, is zero.

'The first two statements have the following global analogs:

(1') $A^{2}(X)$ is finite-dimensional [13].

(2) $A^{2}(X)=\mathbb{Z} \times \operatorname{Alb}_{X}(k)(k=$ base field $)$.

These latter are statements about the Chow group of zero-cycles on $X$, i.e., about the rational points of the functor $\mathscr{L}^{2}(X)$. Roitman demonstrated the equivalence of $\left(1^{\prime}\right)$ and $\left(2^{\prime}\right)$, and Mumford showed that they imply $p_{g}=0$.

Bloch conjectured that $A^{2}(X)$ is finite-dimensional whenever $p_{g}=0$, and proved it for surfaces not of general type [5]; however, his methods yield no grasp of the general situation. 
It seems natural to try:

(A) to define a map of functors $\mathrm{Hilb}_{X}^{\text {cod }=i} \rightarrow \mathscr{A}^{i}(X)$, and

(B) to define a map of functors $\mathscr{L}^{n}(X) \rightarrow \operatorname{Alb}_{X}(n==\operatorname{dim} X)$.

Step A would generalize the cycle map, and would cxplain the rclation between our functor and the concrete notion of relative cycle. In [4] Bloch wondered whether such a map exists and whether its image ever generates $\mathscr{A}^{i}(X)(S)$. In this paper we accomplish step A for infinitesimal points (and much more if $X$ is a curve) by formulating and proving a suitably relative form of Gersten's conjecture (1.3).

It should be remarked that it is easy to define a map $U \rightarrow \mathscr{A}^{i}(X)$ if $U$ is a smooth subscheme of Hilb $_{X}$ by pulling back the canonical class in $H^{i}$ $\left(X \times U, \mathscr{K}_{i}\right)$. This approach is not likely to lead to a computation of $\mathscr{A}^{i}(X)$, however. A consequence of the relative form of Gersten's conjecture over a smooth base $(1.3$, iii) is that the resulting map is the same as ours.

Step B could lead to an alternate proof of Bloch's discovery about the formal completion of $\mathscr{A}^{2}(X)$. His technique is truly mysterious, for the map he defines on formal completions seems to go the wrong way, that is, from $\mathrm{Alb}_{X}$ to $\mathscr{A}^{2}(X)$. We make a good start on step B (and go all the way when $X$ is a curve) by using the notion of linear determinant. The main difficulty encountered is that the categories involved are not Abelian, and thus some powerful theorems do not apply. For instance, there is no notion of quotient category, and thus one cannot show that the sheaves in the resolution of $\mathscr{K}_{n}$ are flasque. We are able to show the first one is flasque (when $S$ is artin) (3.8) by using the localization theorem for projective modules. Thus we can accomplish step B for a curve (for infinitesimal points) and this motivates the construction of a certain non-Abelian quotient category.

A purely algebraic consequence of (1.3) and (3.8), pointed out to me by Spencer Bloch, is the following. If $F$ is the total quotient ring of a local ring $R$ essentially smooth over an artinian ring, then the map $K_{i} R \rightarrow K_{i} F$ is injective.

\section{Notation}

If $X$ is a scheme and $x \in X$, then $X_{x}$ denotes $\operatorname{Spec}\left(\mathcal{O}_{X, x}\right)$, where $\mathscr{O}_{X, x}$ is the local ring at $x$, and $X_{x}^{\text {sh }}$ is the strict henselization.

If $X \rightarrow S$ is a map of schemes, and $s \in S, X(s)$ denotes the fiber $X \otimes \otimes_{S} k(s)$, where $k(s)$ is the residue field of $s$.

If $S$ is a scheme or a ring, $\mathbb{A}_{S}{ }^{n}$ denotes affine $n$-space over $S$.

We use the terminology of $K$-theory and exact categories as developed in [Q].

If $X \rightarrow S$ and $T \rightarrow S$ are maps of schemes, $X_{T}$ denotes the fiber product $X \times{ }_{s} T$. 
If $X$ and $T$ are $S$-schemes, $X(T)$ denotes the set of maps from $T$ to $X$ over $S$. We also use this notation when $T$ is a ring.

All schemes are assumed to be locally Noetherian, and all maps are separated.

\section{The Relative Situation}

In this section we define the exact categories we will need, and we present our form of Gersten's conjecture.

Definition. If $X$ is an $S$-scheme flat over $S$, let $\mathscr{M}^{p}(X / S)$ denote the exact category of quasi-coherent sheaves of finite type on $X$ which are flat over $S$ and have relative codimension $\geqslant p$.

Propostrion 1.1. Suppose $f: X \rightarrow Y$ is a map of flat $S$-schemes; then pullback defines an exact functor $\mathscr{M}^{p}(Y / S) \rightarrow \mathscr{M}^{p}(X / S)$ if $f$ is flat, and direct image defines an exact functor $\mathscr{M}^{p}(X / S) \rightarrow \mathscr{M}^{p}(Y / S)$ if $f$ is finite. Moreover, pullback along a map $T \rightarrow S$ defines an exact functor $\mathscr{M}^{p}(X / S) \rightarrow \mathscr{M}^{p}\left(X_{T} / T\right)$.

Proof. The statements are local, and thus we may assume the schemes involved are affine. In this case it is clear that the various functors involved preserve codimension.

Q.E.D.

Proposition 1.2. Suppose X/S is of finite type and $x \in X$. Then

$$
K_{*}\left(\mathscr{M}^{p}\left(X_{x} / S\right)=\varliminf K_{*}\left(\mathscr{M}^{p}(U / S)\right),\right.
$$

where $U$ ranges over all open neighborhoods of $x$. The analogous statement for $X_{x}^{\text {sh }}$ holds as well.

Proof. One reasons as in [15, Sect. 7, Proposition 2.2]. The only problem is to see that each flat module on $X_{x}$ (resp. $X_{x}^{\text {sh }}$ ) extends to a flat module on some neighborhood $U$ (resp. some etale neighborhood). Since $X / S$ is of finite type, flatness is an open property on $X$, and this takes care of the first case. In the etale situation, each flat module on $X_{x}^{\mathrm{sh}}$ comes from some module $M$ on an etale neighborhood $U$ of $x ; M_{x}$ is flat because $X_{x}^{\text {sh }} \rightarrow U_{x}$ is faithfully flat, and thus $M$ is flat in an open neighborhood of $x$ in $U$.

Definition. Let $K_{q}{ }^{p}(X / S)$ denote $K_{q}\left(\mathscr{M}^{p}(X / S)\right)$.

ThEOREM 1.3. Suppose $f: X \rightarrow S$ is a smooth map of schemes, $x \in X$, and $p \geqslant 0$. If one of the following conditions holds:

(i) $S$ is the spectrum of an artin local ring, 
(ii) $S$ is the spectrum of a local Noetherian henselian ring, $X / S$ has fibers of dimension 1 , and $x$ is in the closed fiber, or

(iii) $S$ is a smooth variety over a field $k$, then the map

$$
K_{*}^{p+1}\left(X_{x} / S\right) \rightarrow K_{*}^{p}\left(X_{x} / S\right)
$$

is zero.

Proof. First we consider the case where (i) or (ii) holds. Wc assume $X$ is affine and let $s$ denote the closed point of $S$. Given $N \in \mathscr{M}^{p+1}\left(X_{x} / S\right)$, its support does not contain any generic point of the closed fiber, so we may choose a function $f$ on $X$ which is a nonzero divisor on $X(s)$ and annihilates $N$. By the local criterion of flatness [2, VII, 4.1, p. 142], $f$ is a relative nonzero divisor near $x$; this means that $f$ is a nonzero divisor and the subscheme $Z$ defined by $f=0$ is flat over $S$ near $x$-such a $Z$ is called a relative divisor. By (1.2) and the fact that $K *$ commutes with filtering direct limits we see that

$$
K_{*}^{p+1}\left(X_{x} / S\right)=\text { ind. } \lim _{Z \subset X} \operatorname{ind}_{U \subset Z} \lim _{*} K_{*}^{p}(U / S),
$$

where $Z$ runs over increasingly large closed subschemes of $X$ which are relative divisors near $x$, and $U$ runs over increasingly small neighborhoods of $x$ in $Z$ which are flat over $S$. Thus it suffices to show that the various maps $K_{*}^{p}(U / S) \rightarrow$ $K_{*}^{p}\left(X_{x} / S\right)$ are all zero. For this purpose we may replace $X$ by some open subset and achieve $U=Z$. Let $r=\operatorname{dim}(X \mid S)$.

According to Quillen's variant of Noether's normalization lemma [15, Sect. 7, Lemma 5.12], or [11], there is a projection $X(s) \rightarrow \mathbb{A}_{k(s)}^{r-1}$ which is finite on $Z(s)$ and smooth at $x$. (In case (ii), $r=1$ and this map must be the structure map.) It is induced by some projection $p: X \rightarrow \mathbb{A}_{S}^{r-1}$, for $S$ is local. In case (i), $p$ is finite on $Z$ by Nakayama's lemma, and $p$ is smooth at $x$ by [18, II, Corollary 2.2]. In case (ii) we already know $p$ is smooth at $x$; by Hensel's lemma we may replace $X$ by an open neighborhood of $x$, getting rid of components of $Z$ on which $p$ may not be finite, and we may ensure that $p$ is finite on $Z$.

From this point on, the proof continues mutatis mutandis as in [15, Sect. 7, Theorem 5.11] after the proof of Lemma 5.2, except that $k$ is replaced by $S$, and is no longer a field.

(When $S$ is not artin, the problem with the approach above is illustrated by the following example. Let $X$ be a family of surfaces over $S$, let $Z$ be a relative divisor in $X$ with irreducible generic fiber and with a reducible fiber $Z(s)=$ $Z_{1} \cup Z_{2}$ over $s$, let $U=Z-Z_{1}$, and choose $x \in U(s)$. A generic projection will map both $Z_{1}$ and $Z_{2}$ onto $\mathbb{A}_{k(s)}^{r-1}$ and thus will fail to be finite on $U$, although it will be quasi-finite. Henselizing the image of $x$ under the projection will cure this problem but force henselization at $x$. Thus everything works if the etale topology is used, but this fact does not seem useful because we do not know what $H_{\mathrm{et}}^{i}\left(X, \mathscr{K}_{i}\right)$ is when $X$ is a smooth variety. $)$ 
Now suppose (iii) holds. Now $K_{*}^{p+1}\left(X_{x} / S\right)=\underline{\varliminf} K_{*}^{p+1}(U / S)$ where $U$ ranges over open neighborhoods of $x$. Since $X$ is a variety, each $U$ will contain a closed point $y$ in the closure of $x$, and the diagram

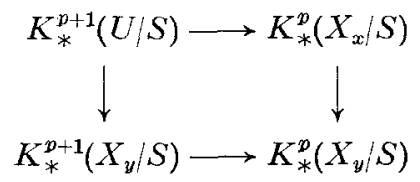

shows that we may as well replace $x$ by $y$ and assume $x$ is a closed point. Given $N \subset \mathscr{M}^{p+1}\left(X_{x} / S\right)$, we may find, as before, a relative divisor $Z^{\prime}$ near $x$ supporting $N$, and since $Z^{\prime}$ is also relatively Cohen-Macaulay, we may continue to whittle it down until we find that $N$ is supported by a subscheme $Z \subset X$ which, near $x$, is relatively regularly immersed and has codimension $p+1$ (i.e., it is defined by a relatively regular sequence $f_{1}, \ldots, f_{p+1}$ of functions on $X$ ). That the system of such subschemes $Z$ is filtering may be seen by using the statement above when $N=\mathcal{O}_{Z_{1}} \oplus \mathcal{O}_{Z_{2}}$. By the same sort of reasoning as before it is enough to show that the map

$$
K_{*}^{0}(Z / S) \rightarrow K_{*}^{p}\left(X_{x} / S\right)
$$

is zero.

Now $\mathcal{O}_{Z}$ is a Cohen-Macaulay $\mathscr{O}_{X}$-module, so for each $N \in \mathscr{M}^{0}(Z / S)$ we may construct a resolution of $N$ by Cohen-Macaulay modules of the same dimension as $Z$. The characterization of depth as the vanishing of Ext groups in low degree [2, III, 3.13, p. 52] yields the following. If $0 \rightarrow M^{\prime} \rightarrow M \rightarrow M^{\prime \prime} \rightarrow 0$ is an exact sequence of finite $\mathcal{O}_{X}$-modules, then

(i) $\operatorname{depth}_{z} M \geqslant r$ and $\operatorname{depth}_{z} M^{\prime \prime} \geqslant r-1$ imply $\operatorname{depth}_{z} M^{\prime} \geqslant r$,

(ii) $\operatorname{depth}_{z} M^{\prime} \geqslant r$ and $\operatorname{depth}_{z} M^{\prime \prime} \geqslant r$ imply $\operatorname{depth}_{z} M \geqslant r$.

These imply that the full subcategory $\mathscr{C} \mathscr{M}$ of $\mathscr{M}^{0}(Z / S)$ consisting of those $M$ with $\operatorname{depth}_{Z} M \geqslant \operatorname{dim} Z$ is an exact category and that each $N$ in $\mathscr{M}^{0}(Z / S)$ has a finite resolution by modules in $\mathscr{C} \mathscr{M}$. (An object of $\mathscr{C} \mathscr{M}$ is either 0 or a CohenMacaulay module of maximal dimension.) The resolution theorem [15, Sect. 4, Corollary 1 to Theorem 3] says that

$$
K_{*} \mathscr{C} \mathscr{M} \rightarrow K_{*}^{0}(Z / S)
$$

is an isomorphism.

We show that

$$
K_{*} \mathscr{C} \mathscr{M} \rightarrow K_{*}^{\nu}\left(X_{x} / S\right)
$$

is zero by projecting sideways, a technique used in [21] and originally suggested by Steve Kleiman. 
Let $r=\operatorname{dim} X, d=\operatorname{dim} Z, a=\operatorname{dim} S$, and $s=f(x) ;$ then $Z(s) \quad \cdots$ $X(x) \cap Z$ has dimension $d-a$. Suppose we can find a projection

$$
\pi: X \rightarrow \mathbb{A}^{r-1}
$$

such that

(1) $\pi$ is smooth at $x$,

(2) $\pi$ is finite on $Z$ and on $X(s)$,

(3) $\operatorname{dim}\left(X(s) \cap \pi^{-1} \pi Z\right) \leqslant d-a+1$.

Given $N \in \mathscr{C} \mathscr{M}$, we see that $\pi_{*} N$ has the same depth and dimension as $N$ [2, III, 3.16, p. 53], so is Cohen-Macaulay.

The fibers of $\pi$ are smooth of dimension 1 near $x$, so have depth $=1$. It follows that depth $\pi^{*} \pi_{*} N=$ depth $\pi_{*} N+1$ near $x$, so $\pi^{*} \pi_{*} N$ is Cohen Macaulay near $x\left[14,21 . \mathrm{C}\right.$, p. 153]. Now hypothesis (3) says that $\pi^{*} \pi_{*} N$ meets the fiber $X(s)$ properly, so $\left(\pi^{*} \pi_{*} N\right)_{x}$ is flat over $S[2, \mathrm{~V}, 3.5, \mathrm{p} .94]$ and thus is in $\mathscr{M}^{p}\left(X_{x} / S\right)$. From this point on, as before, the proof continues as in $[15$, Sect. 7, Theorem 5.11].

Now for the problem of finding $\pi$. If $k$ is finite such $\pi$ may not exist, but the trick of Joel Roberts as explained in [11] shows that we may assume $k$ is infinite. Suppose $X \subseteq \mathbb{A}^{N}$, and consider the family of linear projections $\mathbb{A}^{N} \rightarrow \mathbb{A}^{r-1}$; it is parametrized by an affine space. The generic projection satisfies (1) and (2), as explained in [11], and the following lemma, together with the finiteness of the map $X(s) \cap \pi^{-1} \pi Z \rightarrow \pi X(s) \cap \pi Z$, shows it satisfies (3).

Q.E.D.

Lemma 1.3.1. Suppose $W$ and $Z$ are closed subvarieties of $A^{N}$ of dimensions $d$ and e, respectively. If $\operatorname{dim}(W \cap Z)=d+e-r$ or $W \cap Z=\varnothing$, then a general projection $\pi: \mathbb{A}^{N} \rightarrow \mathbb{A}^{r-1}$ satisfies $\operatorname{dim} \pi W \cap \pi Z \leqslant d+e-r+1$.

Proof. It is enough to show that a general linear projection $\pi: \mathbb{A}^{N} \rightarrow \mathbb{A}^{N-1}$ satisfies

(1) $\operatorname{dim} \pi W \cap \pi Z \leqslant d+e-r+1$ if $r=N$, and

(2) $\operatorname{dim} \pi W \cap \pi Z \leqslant d+e-r$ if $r<N$.

Let $Q==(W-W \cap Z) \times(Z-Z \cap W)$, let $H$ be the hyperplane $\mathbb{F}^{* N}-\mathbb{A}^{N}$, and let $f: Q \rightarrow H$ be the map which associates to $(w, z)$ the point of $H$ which lies on the line through $w$ and $z$. If $x \in H$ is the center of $\pi$, then $\pi W \cap \pi Z=$ $\pi(W \cap Z) \cup \pi \circ p r_{1}\left(f^{-1}(x)\right)$. For general $x$, if $f$ is not dominant, then $f^{-1}(x)$ is empty and we are done; if $f$ is dominant, then $f^{-1}(x)$ has dimension $d+e-N+1$, so $\operatorname{dim} \pi W \cap \pi Z \leqslant \max (d+e-N+1, d+e-r)$, and the latter is the bound we want in each of the above two cases. Q.E.D. 
LEMma 1.3.2. Suppose $A \rightarrow B \rightarrow C$ are maps of Notherian rings, $M$ is a finitely generated $C$-module, and $A \rightarrow B$ is étale. Then $M$ is flat over $B$ if and only if $M$ is flat over $A$.

Proof. Flatness is a local condition, so we may assume all the rings and maps are lucal. Étale maps are unramified, so $m_{A} B=m_{B}$. If $P . \rightarrow M$ is a projective $B$-resolution of $M$, then it is also a flat $A$-resolution of $M$. Since $P_{i} / m_{A} P_{i}=$ $P_{i} / m_{B} P_{i}$, we see that $\operatorname{Tor}_{1}{ }^{A}\left(M, A / m_{A}\right)=\operatorname{Tor}_{1}{ }^{B}\left(M, B / m_{B}\right)$, so we are done by the local criterion of flatness.

Q.E.D.

Corollary 1.3.3. Suppose $f: X \rightarrow S$ is smooth with relative dimension $1, S$ is locally Noetherian, and $x \in X$. Then

$$
K_{*}^{\mathbf{1}}\left(X_{x}^{\mathrm{sh}} / S\right) \rightarrow K_{*}^{0}\left(X_{x}^{\mathrm{sh}} / S\right)
$$

is zero.

Proof. Let $s=f(x)$. The dotted arrow exists in the diagram

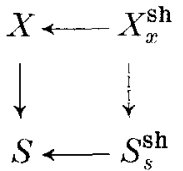

because $X x_{s} S_{s}^{\text {sh }}$ is a limit of étale neighborhoods of $x$ in $X$. According to Lemma (1.3.2), $K_{*}^{p}\left(X_{x}^{\mathrm{sh}} / S\right)=K_{*}^{p}\left(X_{x}^{\mathrm{sh}} / S_{s}^{\mathrm{sh}}\right)$, so we may replace $S$ by $S_{s}^{\mathrm{sh}}$ and $X$ by $X \times{ }_{s} S_{s}^{\text {sh }}$ and assume that $S$ is the spectrum of a local Noetherian henselian ring. Now $X_{x}^{\mathrm{sh}}=\varliminf_{y} Y_{y}$ where the projective limit runs over affine étale neighborhoods $(Y, y)$ of $(X, x)$. Since $K_{*}^{p}\left(X_{x}^{\mathrm{sh}} / S\right)=\underline{\lim } K_{*}^{p}\left(Y_{y} / S\right)$, and the theorem applies to $Y_{y} / S$, we are done.

Q.E.D.

Definition. If $X$ is a scheme, let $\mathscr{P}(X)$ denote the exact category of vector bundles on $X$ (i.e., locally free sheaves of finite rank). Let $K_{q}(X)$ denote $K_{q}(\mathscr{P}(X))$.

Proposition 1.4. If $S$ is affine and Noetherian, and $X$ is a smooth affine S-scheme, then $K_{*}^{0}(X / S)=K *(X)$.

Proof. By the resolution theorem [15, Sect. 4, Corollary 1 to Theorem 3] it is enough to show that any $N$ in $\mathscr{M}^{*}(X / S)$ has a projective resolution $0 \rightarrow P_{n} \rightarrow \cdots \rightarrow P_{0} \rightarrow N \rightarrow 0$, with $n=\operatorname{dim}(X / S)$. Since $X$ is Noetherian and affine we may construct $0 \rightarrow Q \rightarrow P_{n-1} \rightarrow \cdots \rightarrow P_{0} \rightarrow N \rightarrow 0$, an exact sequence where the $P_{i}$ are vector bundles on $X$; we need only show that $Q$ is locally free. Since $Q$ is finitely presented, we may assume that $X$ and $S$ are both local. Let $f$ denote $X \rightarrow S, x$ the closed point of $X$, and $s=f(x)$ the closed point of $S$. We know that $Q$ is flat over $S$ because $N$ and $P_{i}$ are. We also know that $Q(s)$ is locally 
free because $0 \rightarrow Q(s) \rightarrow P_{n-1}(s) \rightarrow \cdots \rightarrow P_{0}(s) \rightarrow N(s) \rightarrow 0$ is exact and $X(s)$ is regular of dimension $\leqslant n$. Since $X$ is flat over $S, Q(s)$ is flat over $X(s)$, and $Q$ is flat over $S$, we conclude that $Q$ is flat over $X$, and thus is locally free. Q.E.D.

The $K$-group $K_{q}\left(\mathscr{M}^{p}(X / S)\right)$ is given by the $(q+1)$ st homotopy group of the topological space $B Q \mathscr{M}^{n}(X / S)$, which is an $H$-space under the operation of direct sum. Consequently, the relative homotopy groups with respect to the subspace $B Q \mathscr{M}^{p+1}(X \mid S)$ are all Abelian groups, and we may make the following definition:

Definition. $\quad K_{q}^{p / p+1}(X / S)=\pi_{q+1}\left(B Q \mathscr{M}^{p}(X / S), B Q \mathscr{M}^{p+1}(X / S)\right)$. The corresponding long exact sequence of homotopy groups has the following form: $\cdots \rightarrow K_{q}^{p+1} \rightarrow K_{q}^{p} \rightarrow K_{q}^{p / p+1} \rightarrow K_{q-1}^{p+1} \rightarrow \cdots$.

COROLlary 1.5. Suppose $X / S$ satisfies the conditions of both (1.4) and (1.3). Then for each $i$ there is an exact sequence $0 \rightarrow K_{i}\left(X_{x}\right) \rightarrow K_{i}^{0 / 1}\left(X_{x} / S\right) \rightarrow$ $K_{i-1}^{1 / 2}\left(X_{x} / S\right) \rightarrow \cdots \rightarrow K_{1}^{i-1 / i}\left(X_{x} / S\right) \rightarrow K_{0}{ }^{i}\left(X_{x} / S\right) \rightarrow 0$.

Definition. Let $\mathscr{K}_{q}^{p / p+1}(X / S)$ denote the sheaf on $X$ associated to the Zariski presheaf $U \vdash K_{q}^{p / p+1}(U / S)$; similarly define $\mathscr{K}_{q}{ }^{p}(X / S)$ and $\mathscr{K}_{q}(X)$. We use the subscript ét to denote the analogous sheaf in the etale topology on $X$.

COROLLARY 1.6. Suppose $X / S$ is smooth and $S$ is artin; then there is an exact sequence of sheaves on $X$ :

$$
0 \times \mathscr{K}_{i}(X)>\mathscr{K}_{i}^{0 / 1}(X / S)>\cdots>\mathscr{K}_{1}^{i-1 / i}(X / S)>\mathscr{K}_{0}^{i}(X / S)>0 .
$$

Proof. We use (1.5) and the fact that $K *$ commutes with filtering direct limits (1.2); note that all the maps in question are defined on the level of presheaves.

Q.E.D.

Corollary 1.6. Suppose $X / S$ is smooth of relative dimension 1 , and $S$ is locally Noetherian. Then there is an exact sequence of sheaves in the étale topology on $X$ :

$$
0 \rightarrow \mathscr{K}_{i}(X)_{\text {et }} \rightarrow \mathscr{K}_{i}^{0 / 1}(X / S)_{\text {ét }} \rightarrow \mathscr{K}_{i-1}^{\mathbf{1}}(X / S)_{\mathrm{et}} \rightarrow 0
$$

\section{The Map from the Hilbert Scheme}

First a few definitions. If $X$ is a scheme, let $A^{i}(X)$ denote $H^{i}\left(X, \mathscr{K}_{i}(X)\right)$. If $X$ is smooth over a field $k$, then $A^{i}(X)$ is the group of codimension $i$ cycles modulo linear equivalence ([15, Sect. 7, Theorem 5.19] or [11]), but otherwise the group $A^{i}(X)$ appears to be mysterious. If $X$ is smooth over a scheme $S$, 
the goal is to represent $A^{i}(X)$ as a group of relative cycles modulo some equivalence. A first step in this direction would be to define a map from the Hilbert scheme to the relativization of this group. If $X$ is a $k$-scheme, we let $\mathscr{A}^{i}(X)$ denote the functor $S \mapsto A^{i}\left(X_{S}\right)$. When $X$ is smooth over $k$, the rational points of this functor constitute the group of codimension $i$ cycles modulo rational equivalence.

The functor $\mathscr{A}^{1}(X)$ is the absolute Picard functor, $S \mapsto \operatorname{Pic}\left(X_{S}\right)$, because $\mathscr{K}_{1}$ is the sheaf of units.

If $X$ is an algebraic $k$-scheme, we let $\operatorname{Hilb}_{X}^{\text {eod }=i}$ denote the part of the Hilbert scheme which parametrizes subschemes of $X_{S}$ proper and flat over $S$ with relative codimension $i$. For a discussion of the representability of $\mathrm{Hilb}_{X}$, see [8].

For the rest of the section we fix a variety $X$ of dimension $n$ which is smooth over a field $k$. If $S$ is artin or $S$ is a smooth variety, then $X_{S} / S$ satisfies the conditions of (1.3), and we can define a map $\operatorname{IIllb}_{X}^{\text {cod }=i}(S) \rightarrow \mathscr{L}^{i}(X)(S)$ by composing the following sequence of maps:

$$
\begin{aligned}
& \operatorname{Hilb}_{X}^{\text {cod }-i}(S) \stackrel{b}{\longrightarrow} K_{0}^{i}\left(X_{S} / S\right) \longrightarrow H^{0}\left(X_{S}, \mathscr{K}_{0}^{i}\left(X_{S} / S\right)\right) \\
& \quad \stackrel{a_{0}}{\longrightarrow} H^{1}\left(X_{S}, \mathscr{K}_{1}^{i-1}\left(X_{S} / S\right)\right) \stackrel{d_{1}}{\longrightarrow} \cdots \stackrel{d_{i-1}}{\longrightarrow} H^{i}\left(X_{S}, \mathscr{K}_{i}^{0}\left(X_{S} / S\right)\right) \\
& =H^{i}\left(X_{S}, \mathscr{K}_{i}\left(X_{S}\right)\right)=\mathscr{A}^{i}(X)(S) .
\end{aligned}
$$

The map $b$ is $Z \mapsto\left[\mathscr{O}_{Z}\right]$, and the map $d_{p-1}$ is a boundary map in the long exact sequence of cohomology groups for the following short exact sequence:

$$
0 \rightarrow \mathscr{K}_{p}^{i-p} \rightarrow \mathscr{K}_{p}^{i-p / i-p+1} \rightarrow \mathscr{K}_{p=1}^{i-p+1} \rightarrow 0 .
$$

Naturality with respect to $S$ is clear. In particular, if $S^{\prime} \rightarrow S$ is a map where $S^{\prime}$ is artin and $S$ is a smooth variety, we get a commutative square:

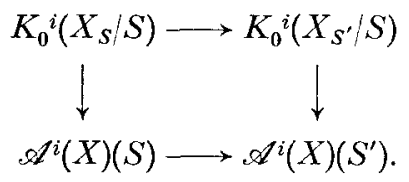

This might justify the inclusion of the case where $S$ is smooth in (1.3), in spite of the fact that $X_{S}$ is a smooth variety and the map from the Hilbert scheme can be defined using Quillen's work alone.

Now suppose $X$ is a curve, so $n=1$. In this case any locally Noetherian $S$ will work because we can use the etale topology. Let $A_{\mathrm{ei}}^{n}(X)$ denote the étale cohomology group $H_{\mathrm{et}}^{n}\left(X, \mathscr{K}_{n}\right)$, and let $\mathscr{A}_{\mathrm{et}}^{n}(X)$ denote the functor $S \mapsto$ $A_{\mathrm{et}}^{n}(X \times S)$. Since $\mathscr{A}_{\mathrm{et}}^{1}(X)=\mathscr{A}^{1}(X)$, we can use (1.6) to obtain the map Hilb $_{X}^{\text {cod }=1}(S) \rightarrow \mathscr{A}^{1}(X)(S)$ for any locally Noetherian $S$, and the map will be natural in $S$. 


\section{The Map to the Albanese}

In this section we make an attempt to define a map from $\mathscr{A}^{n}(X)$ to $\mathrm{Alb}_{X}$ when $n=\operatorname{dim} X$. We describe the linear determinant [8, pp. 221-225] or [12]), and use it to define a map from $K_{0}{ }^{F}\left(X_{S} / S\right)$ to $\operatorname{Alb}_{X}(S)$. When $S=\operatorname{Spec}(k)$, this process is the same as adding up the points in the support of $M$ according to the group law in the Albanese. For information about the Albenese variety, see [17]. We formulate a definition of linear equivalence which behaves well with respect to this map, but are unable to show that the sheaves $\mathscr{K}_{i}^{n-i / n-i+1}$ are flasque for $i>0$. Our best result in this direction is (3.8), which identifies $\mathscr{K}_{i}^{0 / 1}$, the first term in the resolution of $\mathscr{K}_{i}$. It follows that this sheaf is locally constant when $S$ is artin, and we obtain a presentation of the Picard group $A^{1}\left(X_{S}\right)$ in (3.9). When $n=1$, this allows us to define the desired map (3.13).

Since the affine case is so much simpler, we describe it first. The setup is

$$
\begin{aligned}
& X=\operatorname{Spec}(R) \text { is a commutative group scheme over } S, \\
& S=\operatorname{Spec}(A), \\
& h_{n}: R \rightarrow R \otimes_{A} \cdots \otimes_{A} R \text { is the multiplication map, } \\
& h_{0}: R \rightarrow A \text { is the identity of the group, and } \\
& + \text { denotes the group operation. }
\end{aligned}
$$

The $h_{n}$ are well defined by the associativity of the group, and the commutativity of the group means that $h_{2}=s \circ h_{2}$, where $s$ is the switch isomorphism of $R \otimes{ }_{A} R$.

Whenever $N_{1}, \ldots, N_{t}$ are $R$-modules, we consider the tensor product $N_{1} \otimes_{A} \cdots \otimes_{A} N_{t}$ to be an $R$-module via $h_{t}$. The commutativity of the group then allows us to treat $A_{A}{ }^{k} N$ as an $R$-module via $h_{k}$. If $N$ is projective of rank $k$ as $A$-module, then $A_{A}{ }^{k} N$ is invertible, and thus the composite

$$
A \rightarrow R \rightarrow \operatorname{End}_{A}\left(\Lambda_{A}^{k} N\right)
$$

is an isomorphism. In this way we obtain a section of $R / A$ which we denote $g_{N}$.

Proposition 3.1. Suppose $0 \rightarrow M \rightarrow N \rightarrow P \rightarrow 0$ is an exact sequence of $R$-modules which are projective and of finite rank over $A$. Then $g_{N}=g_{M}+g_{P}$ holds in the group $X(S)$.

Proof. Let det $N$ denote the highest exterior power of $N$. Over $A$ we have an isomorphism

$$
\operatorname{det} M\left(\otimes_{A} \operatorname{det} P=\operatorname{det} N\right.
$$

According to our conventions both sides are also $R$-modules, the left via $\left(h_{m} \otimes h_{p}\right) \circ h_{2}$, and the right via $h_{n}$, where $m, n, p$ are the ranks of $M, N, P$. 
Associativity of the group amounts to the formula that $h_{n}=\left(h_{m} \otimes h_{p}\right) \circ h_{2}$, so this isomorphism is $R$-linear, and the result is immediate from this. Q.E.D.

Definition. Given any $X / S$, let $\mathscr{M}^{F}(X / S)$ denote the exact category of coherent sheaves on $X$ which are flat over $S$ and have support finite nver $S$. We also let $K_{q}{ }^{F}(X / S)$ denote $K_{q}\left(\mathscr{M}^{F}(X / S)\right)$.

The proof that $\mathscr{M}^{F}(X / S)$ is an exact category is postponed until (3.3.1).

Notc now that $\mathscr{H}^{F}(X / S)=\mathscr{M}^{F}(R / A)$ is the category of $R$-modules which are projective and of finite rank over $A$. Thus (3.1) says we can define a map $K_{0}^{F}(X / S) \rightarrow X(S)$ by sending $N$ to $g_{N}$.

Note. The group $K_{0}{ }^{F}(X / S)$, with $\lambda$-operations defined using the exterior powers, and product defined using $h_{2}$ and tensor product, as above, is a $\lambda$-ring.

We now remove the hypothesis that $X$ is affine. The situation is:

$X$ is a commutative group scheme of finite type over $S=\operatorname{Spec}(A)$, $h_{n}: X \times_{s} \cdots \times_{s} X \rightarrow X$ is the multiplication map, and + denotes the group operation.

Whenever $N_{1}, \ldots, N_{t}$ are coherent sheaves on $X$, we may form the $\mathcal{O}_{X}$-module $h_{t *}\left(N_{1} \otimes \otimes_{A} \cdots \otimes_{A} N_{t}\right)$. To construct det $N$ we use the following artifice: Given $N$ in $M^{F}(X / S)$ let $B$ be a coherent $A$-algebra and $\operatorname{Spec}(B) \subseteq X$ be a closed immersion so that $N$ lives on $B$. Let $n=\operatorname{rank} N$, and let $B_{n} \subseteq B\left(\otimes_{A} \cdots\left(\otimes_{A} B\right.\right.$ be the subring of symmetric tensors of degree $n$. By [19,3, V, Theorem 4.1] $\operatorname{Spec}\left(B_{n}\right)$ is the quotient of $\operatorname{Spec}\left(B \otimes_{A} \cdots \otimes_{A} B\right)$ by the symmetric group, and we get the arrow $q$ in the following diagram because the group $X$ is commutative:

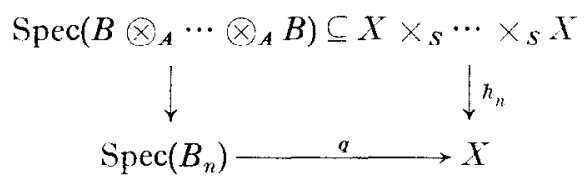

Definition. $\operatorname{det} N=q_{*}\left(A_{A}{ }^{n} N\right)$, where $A_{A}{ }^{n} N$ is considered to be a $B_{n^{-}}$ module.

We see easily that det $N$ does not depend on the choice of $B$, and is invertible as $A$-module. Thus, as before, we may use $\operatorname{det} N$ to determine a section $g_{N}$ of $X / S$.

Proposition 3.2. Suppose $0 \rightarrow M \rightarrow N \rightarrow P \rightarrow 0$ is an exact sequence in $\mathscr{M}^{F}(X / S)$. Then $g_{N}=g_{M}+g_{P}$.

Proof. Choose a closed subscheme $\operatorname{Spec}(B) \subseteq G$ on which $N, M$, and $P$ all live. As in (3.1) it is enough to show that $\operatorname{det} N=h_{2 *}\left(\operatorname{det} M \otimes_{A} \operatorname{det} P\right)$. We may 
assume $M, N$, and $P$ have constant ranks $m, n$, and $p$ over $A$. Consider the situation

$$
\begin{gathered}
\Lambda^{m} M \otimes_{A} \Lambda^{p} P \\
\vdots \\
B_{n} \subset \longrightarrow B_{m, p} \longleftarrow B_{m} \otimes_{A} B_{p}
\end{gathered}
$$

where $B_{m, p}$ is the invariant subring of $B^{\otimes n}$ under the group $S_{m} \times S_{p}\left(S_{m}=\right.$ the symmetric group). Since we can make $B_{m, p}$ act on $\Lambda^{m} M \otimes \Lambda^{p} P$, we find an isomorphism of $B_{n}$-modules: $\Lambda^{n} N \cong A^{m} M \otimes_{A} A^{p} P$. The commutativity of the following diagram shows that we get the same answer in $X$ :

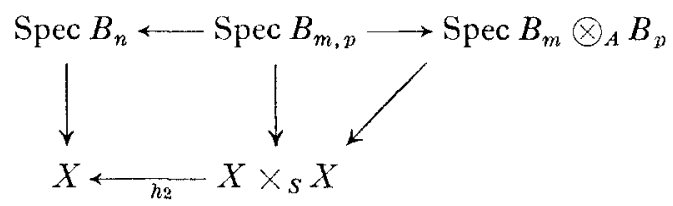

Q.E.D.

Proposition 3.3. Suppose $f: X \rightarrow Y$ is a map of $S$-schemes. The direct image $f_{*}$ is an exact functor $\mathscr{M}^{F}(X / S) \rightarrow \mathscr{M}^{F}(Y / S)$.

Proof. Given $N$ in $\mathscr{M}^{F}(X / S)$, we show $f_{*} N \in \mathscr{M}^{F}(Y / S)$; exactness follows easily. We may assume $X=\operatorname{Supp} M$ and $X$ is finite over $S$. Since $Y / S$ is separated and $X / S$ is proper, $f$ is proper. Since $X / S$ is finite, it follows that $f$ is quasi-finite, and by Chevalley's finiteness theorem $f$ is finite. It follows that $f_{*} N \in \mathscr{M}(Y / S)$ and its support is $f(X)$. We may assume $f(X)=Y$; then $f$ is surjective, thus universally surjective, and this with the finiteness of $X / S$ shows that $Y$ is proper over $S$ with finite fibers. By Chevalley's theorem $Y$ is finite over $S$.

Q.E.D.

COROLlaRy 3.3.1. $\mathscr{M}^{F}(X / S)$ is closed under extension in $\mathscr{M}(X / S)$, and is thus an exact category.

Proof. Given an exact sequence $0 \rightarrow N^{\prime} \rightarrow N \rightarrow N^{\prime \prime} \rightarrow 0$ with $N^{\prime}$, $N^{\prime \prime} \in \mathscr{M}^{F}(X \mid S)$, we must show $\operatorname{Supp}(N)$ is finite over $S$. We may assume the sequence splits because $\operatorname{Supp}(N)=\operatorname{Supp}\left(N^{\prime} \oplus N^{\prime \prime}\right)$. Let $M$ be the coherent sheaf on $Z=X \amalg X$ which is $N^{\prime}$ on one piece and $N^{\prime \prime}$ on the other, and let $f: Z \rightarrow X$ be the diagonal map. Thus $f_{*} M=N$.

Q.E.D.

The most general situation in which we can define the linear determinant is now the following:

$X$ is a scheme over $S$,

$G$ is a commutative group scheme over $S$, and

$g: X \rightarrow G$ is a map of $S$-schemes. 
We define the linear determinant to be the map obtained by composing the following maps:

$$
K_{0} F(X / S) \rightarrow K_{0} F(G / S) \rightarrow G(S)
$$

Since the construction of the linear determinant is local on $S$, we no longer require $S$ to be affine.

The following proposition tells us that the linear determinant is the right way of adding (according to the group law in $G$ ) points from the support of a module (with multiplicity).

Proposition 3.4. Suppose $S$ is a scheme, $s$ is a geometric point of $S$, and $N$ is in $\mathscr{M}^{F}(X / S)$. Then $g_{N}(s)$ is the point of $G(s)$ given by the expression:

$$
\sum\left(\text { length }_{x} N(s)\right) \cdot g(x)
$$

where the sum runs over all rational points $x$ of $X(s)$.

Proof. By compatibility with base-change, we may assume $S$ itself is the spectrum of an algebraically closed field $k$. By additivity we can reduce to the case where $N \cong k(x)=k$. This is the case of rank 1 over $S$, so the linear determinant is obtained from the map:

$$
k \rightarrow O_{G} \rightarrow \operatorname{End}_{k}(k(g(x)))=k .
$$

Note that we have reproved Weil's theorem about correspondences and the maps they induce to the Jacobian of a curve [20, Theorem 22, p. 161].

It is now fairly straightforward to extend our definition of linear determinant to $H^{0}\left(X, \mathscr{K}_{0}^{n}(X / S)\right)$, where $n=\operatorname{dim}(X / S)$, as we shall now see.

Proposition 3.5. Suppose $X / S$ satisfies either (i) $X / S$ is flat, proper, and has relative dimension $n$, or (ii) $X=Y_{y}$, where $Y / S$ is flat and finite type with relative dimension $n$ at $y$ in $Y$, and $S$ is local henselian. Then $\mathscr{M}^{n}(X / S)=\mathscr{M}^{F}(X / S)$.

Proof. The statement follows from the Chevalley finiteness theorem or from Hensel's lemma.

Q.E.D.

Proposition 3.6. Suppose $X / S$ is flat, proper, of relative dimension $n$, and $X \rightarrow G$ is a map to a commutative group scheme over $S$. Then there is a unique way to complete the diagram:

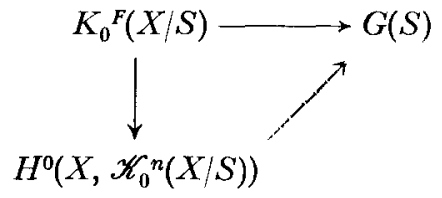

in a way which is natural in $S$. 
Proof. Equip $S$ with the étale topology. Let $\mathscr{R}^{0} \mathscr{K}_{0}{ }^{n}$ be the sheaf associated to $T \mapsto H^{0}\left(X_{T}, \mathscr{K}_{0}{ }^{n}\left(X_{T} / T\right)\right)$, and let $\mathscr{R}^{0} K_{0}{ }^{F}$ be the sheaf associated to $T \mapsto$ $K_{0}{ }^{F}\left(X_{\Gamma} / T\right)$. Since $G$ is a sheaf, we get

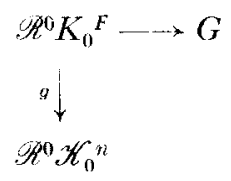

and

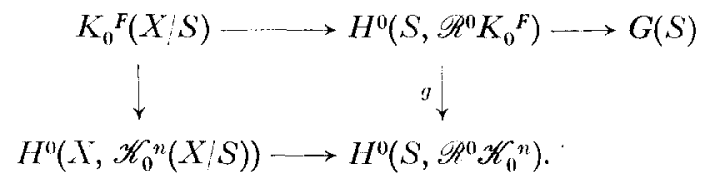

It suffices to show $g$ is an isomorphism, and we do this by checking the stalks in the étale topology on $S$. Since everything commutes with direct limits, we reduce to showing that $K_{0}{ }^{F}(X / S) \rightarrow H^{0}\left(X, \mathscr{K}_{0}{ }^{n}(X / S)\right)$ is an isomorphism when $S$ is henselian. Consider this diagram:

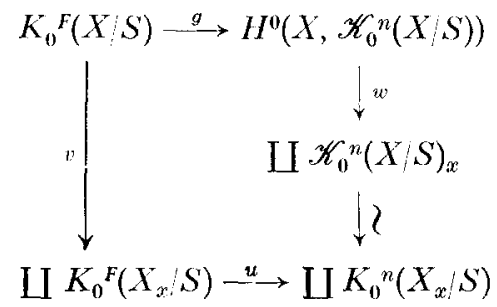

where the sums run over points $x$ in the closed fiber over $S . \mathrm{By}(3.5), u$ is an isomorphism; an application of Hensel's lemma shows that $v$ is an isomorphism. Since $X / S$ is proper, the support of a section of an $\Lambda$ belian sheaf must meet the closed fiber; thus $w$ is injective. Consequently, $w$ and $g$ are isomorphisms.

Q.E.D.

In the classical case, the cycles linearly equivalent to zero map to zero in any Abelian variety. We can define linear equivalence here so that this property still holds.

Definition. If $X$ is a scheme, let $C^{p}(X)$ denote $K_{0}^{p / p+1}(X)$, the group of codimension $p$ cycles on $X$. If $X$ is flat over $S$, let $C^{p}(X / S)$ denote $H^{0}\left(X, \mathscr{K}_{0}^{p / p+1}(X / S)\right)$, and call it the group of relative cycles of codimension $p$ on $X / S$.

Note that if $\operatorname{dim}(X / S)=p$, or $(1.3)$ holds, then $C^{p}(X / S)=H^{0}\left(X, \mathscr{K}_{0} p(X / S)\right)$. 
Also, if $S$ is the spectrum of a field, then $C^{p}(X / S)=C^{p}(X)$; this is the classical case where we already have a definition of linear equivalence of cycles.

If $T \rightarrow S$ is a map of schemes, then there is a natural map $C^{p}(X / S) \rightarrow C^{p}\left(X_{T} / T\right)$ given by pull-back; if $z \in C^{p}(X / S)$, let $z_{T}$ denote the pull-back. If $T=\operatorname{Spec}(k(s))$, where $s \in S$, we let $z(s)$ denote the pull-back.

Definition. If $X$ is a variety over a field $k$, and $S$ is a $k$-scheme, we call a cycle $z \in C^{\mu}\left(X_{S} / S\right)$ linearly equivalent to zero if there is a map of $k$-schemes $S \rightarrow V$ and a cycle $z^{\prime} \in C^{p}\left(X_{V} / V\right)$ such that

(i) $V$ is essentially smooth over $k$,

(ii) $\approx \ldots z_{S}^{\prime}$, and

(iii) for all $v \in V$, the cycle $z^{\prime}(v)$ is linearly equivalent to zero (in the classical sense).

If $z_{1}$ and $z_{2}$ are linearly equivalent to zero, then so is $z=z_{1}+z_{2}$, as can be seen by taking $V=V_{1} \times V_{2}$ and $z^{\prime}=\left(z_{1}^{\prime}\right)_{V}+\left(z_{2}^{\prime}\right)_{V}$.

Note that if $S$ is the spectrum of a field, then this definition does not conflict with the classical definition because change of base field sends cycles which are linearly equivalent to zero to cycles which are linearly equivalent to zero.

Our definition of linear equivalence resembles the definition in [7] of the Chow cohomology groups.

When the conditions of (3.6) are satisfied we let $l d$ denote the map $C^{n}(X / S) \rightarrow$ $G(S)$ obtained there.

Proposition 3.7. If $X$ is a complete variety over $k$ of dimension $n, S$ is a $k$-scheme, and $X \rightarrow G$ is a map to an Abelian variety over $k$, then the map ld: $C^{n}\left(X_{S} / S\right) \rightarrow G(S)$ kills all cycles linearly equivalent to zero.

Proof. Given $z \in C^{n}\left(X_{S} / S\right)$ linearly equivalent to zero, we let $z^{\prime}, V$ and $S, V$ be as in the definition. By naturality of $l d$ it is cnough to sce that $l d\left(z^{\prime}\right)=0$ in $G(V)$. If a map from $V$ to $G$ sends all points to the same closed point, then that map is constant because $V$ is reduced. Thus it is enough to show that $l d\left(z^{\prime}(v)\right)=0$ in $G(k(v))$ for any point $v$ in $V$. For this purpose, we may replace $k$ by $k(v)$ and assume $S=\operatorname{Spec}(k)$. The subgroup of cycles in $C^{n}(X)$ which are linearly equivalent to zero is generated by cycles of the form $\left[Z_{0}\right]-\left[Z_{1}\right]$, where $Z$ is a prime cycle in $X \times A^{1}$ (i.e., subvariety) of codimension $n$ whose intersections with $X \times 0$ and $X \times 1$ are proper, and where $Z_{i}=Z \cdot(X \times i)$ for $i=0,1$. If $Z_{0}$ or $Z_{1}$ is nonempty, then the projection $Z \rightarrow A^{1}$ is dominating, and thus flat. Therefore $Z$ determines a class $[Z] \in C^{n}\left(X \times \mathbb{A}^{1} / \mathbb{A}^{1}\right)$, and $l d(Z)$ is a map $\mathbb{A}^{1} \rightarrow G$. Since $G$ is an Abelian variety, $\operatorname{ld}(Z)$ is constant, so $\operatorname{ld}\left(\left[Z_{0}\right]-\left[Z_{1}\right]\right)=$ $l d(Z) \circ$ inc $_{0}-l d(Z) \circ$ inc $_{1}=0$, where inc and inc $c_{1}$ are the two inclusions of $\operatorname{Spec}(k)$ in $A^{1}$.

Q.E.D. 
Suppose now that $k$ is a field, $X / k$ is a smooth proper variety, $n=\operatorname{dim}(X)$, Alb is the Albanese variety of $X$, and either (i) $S$ is infinitesimal, or (ii) $S$ is local henselian and $n=1$. Given a choice of canonical map $X \rightarrow$ Alb, and refering to the sequence of maps used in Section 2 to construct the map to the Hilbert scheme, the maps we need are those represented by dotted arrows in

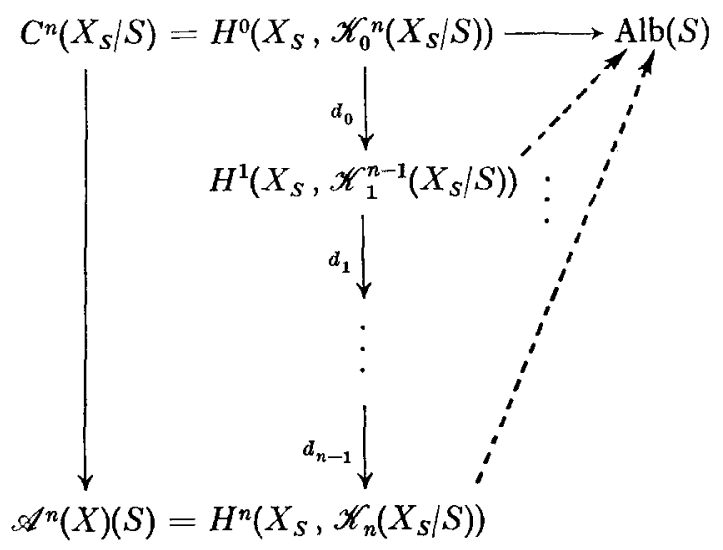

When $S$ is a field, Quillen shows that the sheaves $\mathscr{K}_{j}^{k / k+1}$ are flasque, so $d_{0}$ is surjective, and $d_{1}, \ldots, d_{n-1}$ are isomorphisms; morcover the kerncl of $d_{0}$ consists precisely of the cycles linearly equivalent to zero, and thus dies in $\operatorname{Alb}(S)$. This is what we want to duplicate for any $S$ satisfying the above conditions, but it seems difficult.

We do have the following partial result concerning flasqueness, made possible by the localization theorem for projective modules. Perhaps more could be proved in the presence of more delicate localization theorems. First, a definition: If $R$ is a ring, let $k(R)$ denote the localization of $R$ with respect to the nonzero divisors, and if $R$ is a flat $A$-algebra let $k(R / A)$ denote the localization with respect to the relative nonzero divisors (i.e., the nonzero divisors $f \in R$ such that $R / f R$ is flat over $A$ ). Sheafifying yields sheaves $k(X)$ and $k(X / S)$ for schemes $X$ and $S$.

Theorem 3.8. Suppose $X \mid S$ is smooth, $S$ is locally Noetherian. Then $\mathscr{K}_{i}^{0 / 1}(X / S)$ is the sheaf $\mathscr{K}_{i}(k(X / S))$ associated to the presheaf $U \mapsto K_{i}(k(U / S))$, and is locally constant provided $S$ is finite.

Proof. 'I 'he isomorphism we are about to construct is compatible with localization, so we may assume $X$ and $S$ are affine. Let $X=\operatorname{Spec}(R), S=$ $\operatorname{Spec}(A)$, and let $T \subset R$ be the set of relative nonzero divisors. Let $\mathscr{H}_{R, T}^{1}$ denote the exact category of finitely generated $R$-modules of projective dimension $\leqslant 1$ 
which are killed by $T$, and let $\mathscr{P}^{*}$ be the exact category of $T^{-1} R$-modules of the form $T^{-1} P$ for some $P$ in $\mathscr{P}(R)$. Consider the following diagram:

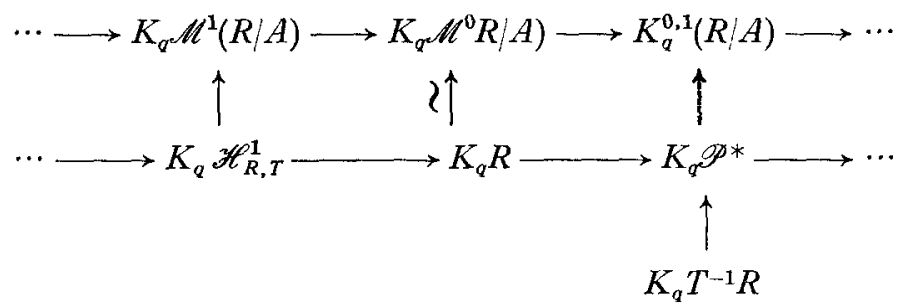

The top row is the long exact sequence resulting from the definition of $K_{q}^{0 / 1}$, and the bottom row is the long exact sequence from the localization theorem for projective modules, [9] or [10]. We also know that $K_{\alpha} \not P^{*} \rightarrow K_{q} T^{-1} R$ is bijective for $q>0$ and injective for $q=0$. The proof of (1.4) shows that every $N$ in $\mathscr{M}^{0}(R / A)$ has projective dimension $\leqslant n=\operatorname{dim}(R / A)$. The argument in the proof of (5.1) shows that $\mathscr{H}_{R, T}^{1} \subset \mathscr{M}^{1}(R / A)$ and yields the left-hand vertical arrow in the diagram; the dotted arrow arises from the universal property of homotopy fibers.

Now we miay sheafify the whole diagram. The desired isomorphisms of sheaves will follow from isomorphisms on the stalks, so we assume $R$ and $A$ are local and $A \rightarrow R$ is a local map. The map $K_{0} \mathscr{P}^{*} \rightarrow K_{0} T^{-1} R$ is an isomorphism because $T^{-1} R$ is the local ring of $R$ at the generic point of its closed fiber over $A$. To see that $K_{q} \mathscr{P P}^{*} \rightarrow K_{q}^{0 / 1}(R / A)$ is an isomorphism it suffices to show that $K_{q} \mathscr{H}_{R, T}^{1} \rightarrow K_{q} \mathscr{M}^{1}(R / A)$ is an isomorphism for all $q$. The latter is accomplished with the aid of the resolution theorem [15, Sect. 4]; we claim that every $N$ in $\mathscr{M}^{1}(R / A)$ has a finite resolution by modules in $\mathscr{H}_{R, T}^{1}$. If $N$ is in $\mathscr{M}^{\mathrm{I}}(R / A)$, we saw in the first part of the proof of (1.3) that $N$ is killed by some relative nonzero divisor $f$. There is a surjection $(R / f R)^{m} \rightarrow \rightarrow N$ and $(R / f R)^{m}$ is in $\mathscr{H}_{R, T}^{1}$; the kernel is in $\mathscr{M}^{\mathrm{I}}(R / A)$, so we continue the resolution to the left. Eventually the kernel is of projective dimension 1 over $R$ and in $\mathscr{H}_{R, T}^{1}$.

Suppose $S$ is artin. We may assume $X$ is connected; it follows that the closed fiber of $X \rightarrow S$ is integral. If $f \in R$, then $f$ is either nilpotent or is a relative nonzero divisor. Thus open sets $U \subset X$ of the form $\operatorname{Spec}\left(R_{f}\right)$, where $f \in T$, constitute a basis for the topology on $X$. The relative nonzero divisors of $R_{f}$ are those of the form $g / f^{m}$, where $g \in T$, so $k(R / A)=T^{-1} R=T^{-1} R_{f}=k\left(R_{f} / A\right)$. It follows easily that the sheaves $k(X / S)$ and $\mathscr{K}_{i}(k(X / S))$ are constant.

Q.E.D.

\section{COROLlaRy 3.9. There is an exact sequence}

$$
H^{0}\left(X, k(X / S)^{*}\right) \rightarrow H^{0}\left(X, \mathscr{K}_{0}^{1}(X / S)\right) \rightarrow H^{1}\left(X, \mathcal{O}_{X}^{*}\right) \rightarrow 0
$$

if $S$ is artin. 
Proof. In the notation of the above proof, $T^{-1} R$ is a local artin ring, so $K_{1}(k(R / A))=k(R / A)^{*}$. The rest follows from the theorem and (1.6). Q.E.D.

Definition. Let $k(X / S)$ denote $H^{0}(X, k(X / S))$ and let div denote the map $k(X / S)^{*} \rightarrow H^{0}\left(X, \mathscr{K}_{0}^{1}(X / S)\right)$.

The map div comes from a boundary map in $K$-theory by sheafification and application of $H^{0}$. Thus, we can compute it explicitly in terms of the following:

Proposition 3.10. Suppose the hypotheses of (3.8) hold and $X:=\operatorname{Spec}(R)$ is affine. Then the map $k(X / S)^{*} \rightarrow K_{0}{ }^{1}(X / S)$ sends $f / g$ to $[R / f R]-[R / g R]$ (for $f, g \in R)$.

Proof. Let $T$ denote the relative nonzero divisors in $R$. Then $f$ and $g$ both divide elements of $T$, and are thus themselves in $T$, as they must be nonzero divisors on each fiber. The result now follows from the last proposition in [10].

Q.E.D.

CoRollary 3.11. If $S$ is the spectrum of a field, then div is the usual map which assigns to a function its divisor.

THEOREM 3.12. Suppose $X$ is a complete nonsingular variety over a field $k$, and $S$ is a finite $k$-scheme. Then the image of the map div: $k\left(X_{S} / S\right)^{*} \rightarrow C^{\mathrm{l}}\left(X_{S} / S\right)$ consists of cycles linearly equivalent to zero.

Proof. We may assume $S$ is local artinian, with one point $s$, and embed it in an affine space $W=A^{m}$. Let $f$ be an element of $k\left(X_{S} / S\right)^{*}$, and let $x$ be the generic point of $X$. Since $k\left(X_{S} / S\right)^{*}$, and let $x$ be the generic point of $X$. Since $k\left(X_{S} / S\right)=\mathcal{O}_{X_{S}, x}$, we may lift $f$ to an element $g$ of $\mathcal{O}_{X_{W}, w}^{*}$. Thinking of $g$ as a global section of the sheaf $k\left(X_{W}\right)^{*}$, we let $g_{y}$ denote the germ at a point $y$. Observe that $k\left(X_{W} / W\right)^{*}$ is a subsheaf of $k\left(X_{W}\right)^{*}$. Since flatness is an open property on $X$, so is the condition that a function be a relative nonzero divisor; it follows that the stalk of the sheaf $k\left(X_{W} / W\right)^{*}$ at $y$ is $k\left(X_{W, y} / W\right)^{*}$.

We claim that for each $y \in X_{W}(s)=X_{S}(s)$, the germ $g_{y}$ is in $k\left(X_{W, y} / W\right)^{*}$. For this purpose, we may assume $X$ is affine and express $g$ as $b / c$, wherc $b$ and $c$ are functions on $X_{W}$ which do not vanish at $x$. By the local criterion of flatness [2, VII, 4.1, p. 142] $b$ and $c$ are relative nonzero divisors near $y$. Thus, $g_{y} \in k\left(X_{W, y} / W\right)^{*}$.

Let $Z$ be the closed subset of $X_{W}$ consisting of all points where $g$ is not in $k\left(X_{W} / W\right)^{*}$. Since $X_{W}$ is proper over $W$, the image of $Z$ in $W$ is closed, and it does not contain $s$. Let $V$ be $W_{s}$. It follows that $h=g_{V}$ is an element of $k\left(X_{V} / V\right)^{*}$.

Now $V$ contains $S$ as well, and $h_{S}=f$. By naturality of the map div, we see that $\operatorname{div}(f)=\operatorname{div}(h)_{S}$. If $v \in V$, then $\operatorname{div}(h)(v)=\operatorname{div}(h(v))$ is the divisor of a function, and is thus linearly equivalent to zero in the classical sense.

Q.E.D. 
Combining this theorem with (3.9) and (3.7) yields the following.

COROLLARY 3.13. Suppose $X$ is a complete nonsingular curve and $J$ is the $J a c o b i a n$ of $X$. If $S$ is an artinian $k$-scheme then the linear determinant map factors through $\mathscr{A}^{1}(X)(S)$ to yield a map $\mathscr{A}^{1}(X)(S) \rightarrow J(S)$.

The following theorem incorporates a suggestion of Steve Kleiman.

THeOREM 3.13. Suppose $X$ is a complete nonsingular curve over a field $k$ and $X \rightarrow G$ is a map to an Abelian variety $G$. If $S$ is a locally Notherian $k$-scheme, then the composite

$$
H^{0}\left(X_{S}, \mathscr{K}_{1}^{0 / 1}\left(X_{S} / S\right)\right) \underset{\mathrm{div}}{\longrightarrow} C^{1}\left(X_{S} / S\right) \underset{l d}{\longrightarrow} G(S)
$$

is zero.

Proof. A map $S \rightarrow G$ is zero if its restriction to any finite subscheme is zero, so we may assume $S$ is finite and apply (3.7) and (3.12). Q.E.D.

\section{Quotient Categories}

Suppose $\mathscr{N} \subseteq \mathscr{M}$ are exact categories. It would be nice if there were an exact category $\mathscr{M} \mid \mathscr{N}$ and an exact functor $\mathscr{M} \rightarrow \mathscr{M} \mid \mathscr{N}$ which killed $\mathscr{N}$, such that the following sequence were exact:

$$
\cdots \rightarrow K_{q+1} \mathscr{M} \mid \mathscr{N} \rightarrow K_{q} \mathscr{N} \rightarrow K_{q} \mathscr{M} \rightarrow K_{q} \mathscr{M} / \mathscr{N} \rightarrow \cdots
$$

Unfortunately this has only been accomplished for Serre subcategories of Abelian categories [15]. It would be nice if we could do this for the categories $M^{p+1}(X / S) \subseteq M^{p}(X / S)$ of Section 1. Theorem 3.8 is an example of success in this direction; the interesting thing is that $\mathscr{P}(k(R / A))$ is almost a quotient category, in the sense of the following universal property:

Definition. $\mathscr{M} / \mathscr{N}$ is a quotient category if, given an exact functor $\mathscr{M} \rightarrow \mathscr{L}$ which also kills $\mathscr{N}$, there is an exact functor $\mathscr{M} / \mathscr{N} \rightarrow \mathscr{L}$, unique up to unique natural isomorphism, which makes the triangle commute:

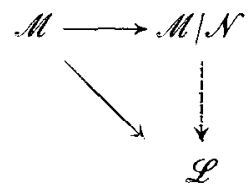


Let $A \rightarrow R$ be a flat local map of local Noetherian rings whose closed fiber is a regular ring of dimension $\leqslant 1$. Let $S$ be the multiplicative subset of $R$ consisting of all the relative nonzero divisors.

LeMma 4.1. $\mathscr{H}^{1}(R / A)=\mathscr{H}_{R, S}^{1}$.

Proof. The proofs of (1.3) and (1.4) show that $\mathscr{H}^{1}(R / A) \subset \mathscr{H}_{R, S}^{1}$. Suppose $M \in \mathscr{H}_{R, S}^{1}$, and $0 \rightarrow P \rightarrow Q \rightarrow M \rightarrow 0$ is a projective resolution of $M$. If $s \in S$ and $s M=0$, then $0 \rightarrow s Q / s P \rightarrow P / s P \rightarrow P / s Q \rightarrow 0$ is exact, and $M \cong s Q / s P$. Then $M$ is flat over $A$ because $P / s P$ is flat and $P / s Q$ has tor-dimension 1; it has codimension 1 because $R / s R$ does.

Q.E.D.

Let $\mathscr{J}$ be the subcategory of $\mathscr{M}^{0}\left(S^{-1} R / A\right)$ whose objects are those of the form $S^{-1} N$ for some $N$ in $\mathscr{M}^{0}(R / A)$.

Proposition 4.2. I is an exact category and is the quotient category of $\mathscr{H}^{0}(R / A)$ by $\mathscr{M}^{1}(R / A)$.

Note. The required long exact sequence of $K$-groups was described in the proof of (3.8). For further details, see [9] or [10].

Proof. By the proof of $(1.4), \mathscr{M}^{0}\left(S^{-1} R / A\right)=\mathscr{P}\left(S^{-1} R\right)$, since $\operatorname{dim}\left(S^{-1} R / A\right)$ 0 ; thus every exact sequence splits in $\mathscr{H}^{0}\left(S^{-1} R / A\right)$ and $\mathscr{J}$ is closed under extensions. 'Thus $\mathcal{J}$ is an exact category.

Let $F: \mathscr{M}^{0}(R / A) \rightarrow \mathscr{L}$ be an exact functor which kills $\mathscr{H}^{1}(R / A)$. Suppose $s \in S$ and $N \in \mathscr{M}^{0}(R / A)$. By the proof of (1.4) there is a projective resolution $0 \rightarrow P \rightarrow Q \rightarrow N \rightarrow 0$. Since $0 \rightarrow P \rightarrow P \rightarrow P / s P \rightarrow 0$ is exact and $F(P / s P)==0$, we see that $F s: F P \rightarrow F P$ is an isomorphism; similarly for $Q$. From the diagram:

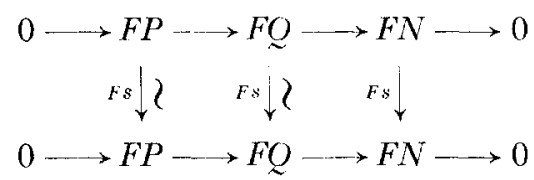

we conclude that $F s: F N \rightarrow F N$ is an isomorphism, and thus $S$ acts invertibly on any $\operatorname{IIom}_{\mathscr{L}}\left(F N^{\prime}, F N\right)$. If $V^{\prime}-S^{-1} N^{\prime}$ and $V=S^{-1} N$, then $\operatorname{Hom}_{S}{ }_{1 R}\left(V^{\prime}, V\right)=$ $S^{-1} \operatorname{Hom}_{R}\left(N^{\prime}, N\right)$, so we get a unique map $\operatorname{Hom}_{S^{-1} R}\left(V^{\prime}, V\right) \rightarrow \operatorname{Hom}_{\mathscr{L}}\left(F N^{\prime}, F N\right)$, and thus an additive functor $\mathscr{J} \rightarrow \mathscr{L}$. It is exact because every exact sequence in $\mathscr{I}$ splits, and the uniqueness is clear.

Q.E.D.

\section{Discussion}

In this section we discuss the sheaf $\mathscr{K}_{0}{ }^{n}(X / S)$ when $n:=\operatorname{dim}(X / S)$ and pursue some suggestions of Spencer Bloch. 
Let $X / k$ be a smooth variety of dimension $n$, let $x \in X$ be a closed point with a regular system of parameters $t_{1}, \ldots, t_{n}$, and suppose $A$ is an artin local ring with residue field $k$ and maximal ideal $\mathbf{m}$. If $k(x)=k$, then $\mathscr{M}^{n}\left(X_{x} \otimes A / A\right)=$ $\mathscr{U}^{F}\left(X_{x} \otimes A / A\right)$ is equivalent to the category $\operatorname{Nil}(n, A)$ whose objects are all $\left(P, f_{1}, \ldots, f_{n}\right)$, where $P \in \mathscr{P}(A)$ and $f_{1}, \ldots, f_{n}$ are commuting nilpotent endomorphisms of $P$.

The Fundamental Theorem [9] says that

$$
\begin{aligned}
\operatorname{ker}\left(K_{0} \operatorname{Nil}(1, A) \rightarrow K_{0} A\right) & =\operatorname{ckr}\left(K_{1} A \rightarrow K_{1} A[T]\right) \\
& =\operatorname{ker}\left(K_{1} A[T] \underset{T \rightarrow 0}{\rightarrow} K_{1} A\right)=N K_{1} A
\end{aligned}
$$

Since $\mathbf{m}$ is nilpotent, we have the following pair of exact sequences.

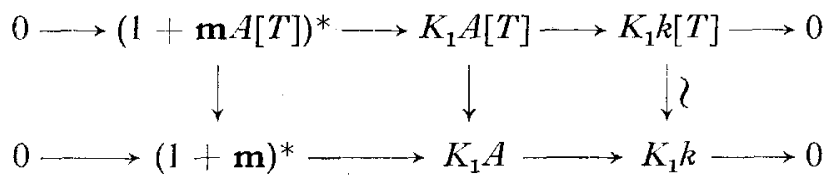

It follows that $N K_{1} A=(1+\mathbf{m} T A[T])^{*}$. The fact that all elements of $N K_{1}$ come from $1 \times 1$ matrices corresponds to the fact that $K_{0}{ }^{1}\left(X_{2} \otimes A / A\right)$ is generated by subschemes $(1.5,3.8,3.10)$. In another paper we will show that $K_{0}{ }^{2}(X \otimes A / A)$ is generated by classes of modules with two generators when $X$ is a surface.

If $A=k[\epsilon] / \epsilon^{2}$, then $N K_{1} A=k \epsilon T+k \epsilon T^{2}+\cdots$, and the term $k \epsilon T$ corresponds to the tangent space $\operatorname{Tan}(X, x)$ when $n=1$ in the following way.

There is a function $s: \operatorname{Tan}(X, x) \rightarrow K_{0}{ }^{F}\left(X_{x} \otimes A / A\right)$ defined by

$$
s(a)=\left[\frac{\mathcal{O}_{x} \otimes A}{t_{1}-a_{1} \epsilon, \ldots, t_{n}-a_{n} \epsilon}\right]-[k(x) \otimes A]
$$

when $a=\sum a_{i}\left(d / \partial t_{i}\right)$. In fact, $s(a)$ is an element of $\operatorname{Tan}\left(\mathscr{C}_{X}{ }^{n}, 0\right)$, where $\mathscr{C}_{X}{ }^{n}$ is the functor $A \mapsto K_{0}{ }^{F}(X \otimes A / A)$. The fact that $s$ is additive for $n=1$ amounts to the existence of the following exact sequences:

$$
\begin{aligned}
& 0 \rightarrow \frac{\mathfrak{C}_{x} \otimes A}{t-\epsilon a} \rightarrow \frac{\mathcal{O}_{x} \otimes A}{(t-\epsilon a)\left(t-\epsilon a^{\prime}\right)} \rightarrow-\frac{\mathcal{Q}_{x} \otimes A}{t-\epsilon a^{\prime}} \rightarrow 0 \\
& 0 \rightarrow-\frac{\bigcup_{x} \otimes A}{t} \rightarrow \frac{\mathscr{O}_{x} \otimes A}{t^{2}-\epsilon\left(a+a^{\prime}\right) t} \rightarrow \frac{\mathscr{O}_{x} \otimes A}{t-\epsilon\left(a+a^{\prime}\right)} \rightarrow 0 .
\end{aligned}
$$

Let $c$ denote the functor $\mathscr{C}_{x^{n}} \rightarrow \mathscr{A}_{X}^{n}$ defined in Section 2 , and suppose that $n=-2$ and $k$ is a number field. It follows from results of [4] that

$$
\begin{aligned}
\operatorname{Tan}\left(\mathscr{A}_{X}^{2}, 0\right) & =H^{2}\left(X, \Omega_{X}^{1}\right)=-\operatorname{Tan}\left(\operatorname{Alb}_{X}, 0\right), \text { and } \\
\operatorname{Bitan}\left(\mathscr{A}_{X}^{2}, 0\right) & =H^{2}\left(X, \mathscr{O}_{X}\right) .
\end{aligned}
$$


The bitangent space of a functor is defined by Bloch to be

$$
\operatorname{Bitan}(F, 0)=\operatorname{ker}\left(F\left(k[\epsilon, \delta] /(\epsilon, \delta)^{2}\right) \rightarrow F^{\prime}\left(k[\epsilon] / \epsilon^{2}\right) \times F\left(k[\delta] / \delta^{2}\right)\right) .
$$

Given $a, b \in \operatorname{Tan}(X, x)$, define $s(a, b) \in \operatorname{Bitan}\left(\mathscr{C}_{X}{ }^{2}, 0\right)$ to be

$$
\begin{aligned}
& {\left[\frac{\mathcal{O}_{x}[\epsilon, \delta]}{t_{1}-a_{1} \epsilon, t_{2}-b_{2} \delta}\right]} \\
& \quad+\left[\frac{\mathcal{O}_{x}[\epsilon, \delta]}{t_{1}-a_{2} \epsilon, t_{2}-b_{1} \delta}\right]-\left[\frac{\mathcal{O}_{x}[\epsilon, \delta]}{t_{1}-a_{1} \epsilon, t_{2}-b_{1} \delta}\right]-\left[\frac{\mathcal{O}_{x}[\epsilon, \delta]}{t_{1}-a_{2} \epsilon, t_{2}-b_{2} \delta}\right] .
\end{aligned}
$$

The elements $c(s(a))$ and $c(s(a, b))$ are probably given by Serre duality and

$$
\begin{aligned}
(\omega \mapsto \omega(a)) \in\left(H^{0} \Omega_{X}\right)^{*} & =H^{2} \Omega_{X}{ }^{1}, \\
(\omega \mapsto \omega(a \otimes b-b \otimes a)) \in\left(H^{0} \Omega_{X}{ }^{2}\right)^{*} & =H^{2} \mathcal{O}_{X} .
\end{aligned}
$$

Note that elements of $H^{2} \Omega_{X}{ }^{1}$ arising this way generate $H^{2} \Omega_{X}{ }^{1}$; this follows from Serre duality and the fact that a differential is determined by its effect on tangent vectors. It follows that the map $\amalg \operatorname{Tan}(X, x) \rightarrow \operatorname{Tan}\left(\operatorname{Alb}_{X}, 0\right)$ (take the image in $\mathrm{Alb}_{X}$ and translate it to 0 ) is surjective, too.

\section{ACKNOWLEDGMENTS}

I thank my advisor Steven Kleiman, for many helpful discussions and ideas, and for his unflagging interest in the problem. I also thank Daniel Quillen, Michael Artin, and Spencer Bloch for many helpful remarks. The contents of this paper are based on my 1976 thesis at MIT, and were partially supported by the NSF.

\section{REFERENCES}

1. M. Artin, Grothendieck 'T'opologies, Notes from Harvard University, Spring, 1962.

2. A. Altman AND S. Klemman, "Introduction to Grothendieck duality theory," Lecture Notes in Math No. 149, Springer-Verlag, Berlin/New York, 1970.

3. S. BLoch, $K_{2}$ and algebraic cycles, Ann. of Math. 99 (1974), 349-379.

4. S. BLoch, $K_{2}$ of artinian $\mathbb{Q}$-algebras, with applications to algebraic cycles, Comm. Algebra 3 (1975), 405-428.

5. S. Bloch, A. Kas, AND D. Lieberman, Zero-cycles on surfaces with $p_{0}=0, \mathrm{Com}$ positio Math. 33 (1976), 135-146.

6. A. Grothendieck and J. Dieudonné, Éléments de géométrie algébrique, Publ. Math. IIIES, Paris.

7. W. Fulton, Rational equivalence on singular varieties, Publ. Math. IHES, No. 45, Bures-sur-Yvette, 1975.

8. A. Grothendieck, "Fondements de la géométrie algébrique," Secrétariat Mathématique, Paris, 1962. 
9. D. Grayson, Higher algebraic K-theory: II (after Daniel Quilen), in "Algebraic $K$-theory," Lecture Notes in Math. No. 551, Springer-Verlag, Berlin/New York, 1976.

10. D. Grayson, The $K$-theory of hereditary categories, J. Pure Appl. Algebra 11 (1977), 67-74.

11. D. Grayson, Projections, cycles, and algebraic K-theory, Math. Ann. 234 (1978), 69-72.

12. B. Iversen, "Linear Determinants with Applications ...," Lecture Notes in Math. No. 174, Springer-Verlag, Berlin/New York, 1970.

13. D. Mumford, Rational equivalence of 0-cycles on surfaces, J. Math. Kyoto Univ. 9 (1969), 195-204.

14. H. Matsumura, "Commutative Algebra," Benjamin, New York, 1970.

15. D. Quru.FN, Higher algebraic $K$-theory: I, in "Algebraic $K$-theory I," Lecture Notes in Math. No. 341, Springer-Verlag, Berlin/New York, 1972.

16. A. Roitman, Rational equivalence of zero-cycles, Math. Sbornik 18 (1972), 571.

17. J.-P. SERRE, Morphisms universeles et variété d'Albanese, Sem. Chevalley, École Normale Supérieur, 1958/59, Exposé 10, Paris.

18. A. Grothendieck, SGA 1: "Revêtements Étales et Groupe Fondamental," Lecture Notes in Math. No. 224, Springer-Verlag, Berlin/New York, 1971.

19. P. Gabricl, "Construction de Preschemas Quotient, Exposé V in SGA 3," Lecture Notes in Math. No. 151, Springer-Verlag, Berlin/New York, 1970.

20. A. WEIL, "Courbes algébriques et variétés abéliennes," Hermann, Paris, 1971.

21. D. Grayson, Products in $K$-theory and intersecting algebraic cycles, Inv. Math. 47 (1978), 71-83. 